\title{
Chronic pulmonary aspergillosis after pulmonary tuberculosis
}

\author{
Edgar Ortiz-Brizuela MD, Alfredo Ponce-de-León MD
}

Cite as: CMAJ 2018 October 1;190:E1171. doi: 10.1503/cmaj.180597

A 68-year-old man who was a farmer from a rural community in Mexico presented to the emergency department with intermittent hemoptysis. He had a history of colon cancer, which had been treated, and pulmonary tuberculosis one year before. Chest tomography showed an irregular spongiform mass in a cavity of the right lung; the cavity had been empty in a radiograph of the chest taken one year earlier (Figure 1A, 1B; a video [Appendix 1] is available at www.cmaj.ca/lookup/suppl/doi:10.1503/cmaj.180597/-/DC1). The main differential diagnoses were malignant disease and tuberculosis reactivation or its sequelae (e.g., bronchiectasis or chronic pulmonary aspergillosis). A video-assisted thoracoscopic segmentectomy was performed; histopathology showed necrotic tissue with hyphae surrounded by a fibrous capsule (Figure 1C). Cultures for bacteria, mycobacteria and fungi grew Aspergillus species only. We diagnosed chronic pulmonary aspergillosis (Figure 1D). Because spillage of the contents of the cavity was not anticipated and did not occur during surgery, we did not administer antifungal therapy. The patient remained asymptomatic after one year of follow-up.

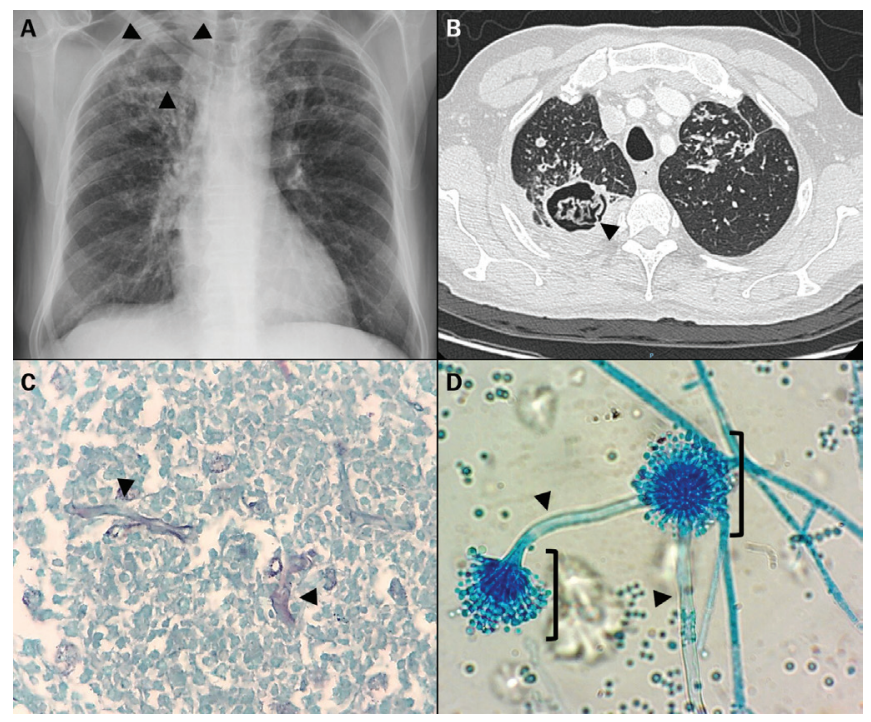

Figure 1: (A) Radiograph of the chest of a 68-year-old man showing an apical empty cavity in the right lung one year before the onset of hemoptysis. (B) Computed tomographic scan of the same cavity in Figure 1A, with an irregular spongiform mass suggestive of a forming aspergilloma. Lung nodules and bronchiectasis are also present. (C) Tissue necrosis and hyphae (arrowheads). Grocott-Gomori methenamine-silver nitrate stain. Original magnification $\times 400$. (D) Conidiophores (arrowheads) and conidial heads (brackets) of Aspergillus species isolated from cultures of surgical specimens. Lactophenol Cotton Blue stain. Original magnification $\times 40$.
Our case highlights the importance of following up patients after treatment of pulmonary tuberculosis. Pulmonary tuberculosis is associated with several pulmonary sequelae; residual cavities are observed in $21 \%-50 \%$ of cases, with chronic pulmonary aspergillosis developing in up to $22 \%$ of cavities. ${ }^{1}$ Although a fungus ball seen on imaging is nearly diagnostic of a single aspergilloma or chronic cavitary pulmonary aspergillosis, ${ }^{2,3}$ serologic or microbiologic evidence of infection with Aspergillus is required for confirmation. ${ }^{3}$

In contrast to a single aspergilloma, chronic cavitary pulmonary aspergillosis is associated with serious symptoms (pulmonary and systemic) and radiographic progression over time., ${ }^{2,3}$ In our patient, the distinction between these entities was not possible given the absence of a surveillance period. Surgery is a definitive therapeutic option for appropriate candidates; antifungal therapy in the perioperative period is only recommended if spillage is anticipated or occurs during the procedure., ${ }^{2,3}$ If surgery is precluded, single aspergilloma requires close monitoring and chronic cavitary pulmonary aspergillosis requires long-term antifungal therapy.,3

\section{References}

1. Denning DW, Pleuvry A, Cole DC. Global burden of chronic pulmonary aspergillosis as a sequel to pulmonary tuberculosis. Bull World Health Organ 2011;89:864-72.

2. Patterson TF, Thompson GR III, Denning DW, et al. Executive summary: practice guidelines for the diagnosis and management of aspergillosis - 2016 update by the Infectious Diseases Society of America. Clin Infect Dis 2016;63:433-42.

3. Denning DW, Cadranel J, Beigelman-Aubry C, et al.; European Society for Clinical Microbiology and Infectious Diseases and European Respiratory Society. Chronic pulmonary aspergillosis: rationale and clinical guidelines for diagnosis and management. Eur Respir J 2016;47:45-68.

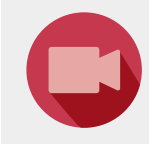

A video of the chest computed tomography scan (coronal reconstruction of the pulmonary window) is available in Appendix 1, at www.cmaj.ca/lookup/suppl/doi:10.1503/ cmaj.180597/-/DC1

\section{Competing interests: None declared.}

This article has been peer reviewed.

The authors have obtained patient consent.

Affiliation: Instituto Nacional de Ciencias Medicas y Nutricion Salvador Zubiran, Vasco de Tlalpan, Mexico City, Mexico

Acknowledgements: The authors thank Andrea Rangel-Cordero and Braulio Martinez-Benitez for providing the microbiology and histopathology images.

Correspondence to: Edgar Ortiz-Brizuela, edgar.brizuela@hotmail.com 